\title{
A fast and simple fluorometric method to detect cell death in 3D intestinal organoids
}

\author{
Konstantin J Bode, ${ }^{1,4}$, Stefanie Mueller', Matthias Schweinlin², Marco Metzger ${ }^{3}$ \& Thomas Brunner*,1
}

\section{ABSTRACT}

Organoids recapitulate the (patho)physiological processes in certain tissues and organs closer than classical cell lines. Therefore, organoid technology offers great potentials in drug development and testing, and personalized medicine. In particular, organoids can be used to study and predict druginduced toxicity in certain tissues. However, until today few methods had been reported to analyze cell death in 3D-microtissues in a quantitative manner. Here, we describe a novel fluorometric method for the quantitative measurement of specific organoid cell death. Organoids are stained simultaneously with the cell impermeable nuclear dye propidium iodide and cell permeable Hoechst33342. While Hoechst allows in-well normalization to cell numbers, propidium iodide detects relative proportion of dead cells independent of hydrogel. Measurement and analysis time, as well as usability are drastically improved in comparison to other established methods. Parallel multiplexing of our method with established assays measuring mitochondrial activity further enhances its applicability in personalized medicine and drug discovery.

\section{METHOD SUMMARY}

We developed a fluorometric method to quantify cell death in intestinal organoids based on DNA staining for normalization and cell permeability for cell death. The method, independent of cell number over a wide range, can be used to study toxic effects of drugs on intestinal organoids or other 3D microtissues, and can be combined with photometric assessment of mitochondrial respiration.

\section{KEYWORDS:}

cell death $\cdot$ DNA dyes $\cdot$ fluorescence $\cdot$ intestine - organoids personalized medicine

'Department of Biology, University of Konstanz, Konstanz, Germany; '2Department of Tissue Engineering \& Regenerative Medicine (TERM), University Hospital Würzburg, Würzburg, Germany; ${ }^{3}$ Translational Centre Regenerative Medicine TLC-RT, Fraunhofer Institute for Silicate Research (ISC), Würzburg, Germany; ${ }^{4} \mathrm{Co}$-operative research training school 'Advanced in vitro test systems for the analysis of cell-chemical interactions in drug discovery \& environmental safety (InViTe)', University of Konstanz, Konstanz, Germany; *Author for correspondence: thomas.brunner@uni-konstanz.de

BioTechniques 67:00-00 (July 2019) 10.2144/btn-2019-0023
Organoids grown from tissue-specific stem cells have become useful tools to study physiological and pathophysiological processes in an in vitro system much closer to the in vivo situation than cell lines. In 2009, Sato et al. described a method on how isolated primary intestinal crypt cells can be cultured over prolonged periods of time [1]. Today, organoids are being generated from nearly every tissue and any organism [2-4]. They are defined as organ-like structures, which self-organize in 3D. Comprised of several tissue- and organspecific cell types, including stem cells, organoids are capable of fulfilling a variety of organ-specific functions, for example, excretion or secretion [5].

Intestinal organoids are being used to study not only basic intestinal physiology [6], but also pathophysiological processes, for example, TNF $\alpha$-induced epithelial cell death during inflammatory bowel disease [7,8]. Moreover, intestinal organoids have been used to study host-pathogen interactions, for example, during Zika virus infection [9]. Importantly, intestinal organoids may also represent an unlimited source of transplantable tissue suitable for regenerative medicine. In a proof-of-principle study murine intestinal organoids were successfully transplanted into the severely damaged colon of mice suffering from inflammatory bowel disease. Organoids not only engrafted functionally into the colon but regenerated the damaged epithelium [10]. Tumor tissue-derived organoids also provide interesting tools to study tumorspecific drug responses as well as tumor diversity ex vivo, for example, in colorectal tumors $[11,12]$. Therefore, primary and tumor organoids are being and will be frequently employed for drug discovery [13] and toxicity testing, but also drug screening in patient-derived malignant tissue [14-16]. In this regard, patient-derived tumor organoid (PDOs) from pancreatic, prostate and gastrointestinal cancer are being used to model drug responses of patientspecific tumors in comparison to normal parental tissue [17-22]. This is of particular interest, as in gastrointestinal cancer it has been demonstrated that the parental and the PDO mutational spectra overlap up to $96 \%$, on top of histological similarities [21].

Thus, by closely resembling the primary tumor in vivo drug testing in PDOs represents a further step towards personalized medicine [21]. Moreover, organoid-like 3D primary cell culture models are being effectively used to screen a large number of emerging oncology compounds for their cytostatic and cell death-promoting activity [23].

A significant problem of cell death screening in organoids is their 3D culture in extracellular matrix, which allows visual qualitative assessment of cell death, but impedes quantitative analysis of cell death. We have previously described the use of a modified MTT staining method to detect organoid survival, specifically, cell death in culture [24]. However, this method is strongly affected by mitochondrial respiration and only an indirect measure of cell survival, specifically, cell death. Other studies have used staining of dying organoids with propidium iodide (PI) and Hoechst33342 (Hoechst) to quantify cell death, employing complex and thus time-consuming high-content imaging [25].

The aim of this study was therefore to develop a simple, practical and quantitative method to study cell death in organoids using PI and Hoechst. While Hoechst is being used to normalize cellularity, PI uptake serves as a measure of cell death. The normalization allows sensitive cell death detection 
over a wide range of cell densities. Fluorescence in 3D cultures can be quantified using a conventional plate reader. The increase of the PI signal relative to the constant Hoechst signal allows calculation of chemotherapeutic drug treatmentspecific organoid cell death. Moreover, this method can be multiplexed with our previously described organoid-optimized MTT assay, allowing simultaneous analysis of respiration/survival and cell death. Thus, in comparison to other known assays our method offers a fast and simple protocol to detect organoid cell death in cell culture plates with minimal computational power and reduced overall costs.

\section{MATERIALS \& METHODS \\ Mice}

C57BL/6 wild-type mice were bred and kept in individually ventilated cages at the central animal facility of the University of Konstanz.

\section{Generation of intestinal organoids}

Intestinal crypts were isolated as described previously with minor changes $[1,24]$. In brief, the small intestine of 8-16-week old C57BL/ 6 wild type mice was cut open longitudinally. Villi were removed by scraping with a microscope slide. Then, the intestine was cut into $3-4 \mathrm{~cm}$ pieces, washed three times with cold $\mathrm{Ca}^{2+}$ - and $\mathrm{Mg}^{2+}$-free PBS, and incubated with $2 \mathrm{mM}$ EDTA in PBS for $30 \mathrm{~min}$ at $4^{\circ} \mathrm{C}$ on a rotating wheel. All subsequent steps until seeding were performed on ice. Supernatant was removed and the tissue was filled up with fresh PBS. After shaking to remove residual villi, fresh PBS was replaced. This step was repeated and each fraction was checked for crypt/villus ratio under the microscope. Up to four crypt-containing fractions were pooled, filtered through a $70-\mu \mathrm{m}$ cell strainer, centrifuged at $100 \times \mathrm{g}\left(3 \mathrm{~min}, 4^{\circ} \mathrm{C}\right)$ and resuspended in $5 \mathrm{ml}$ PBS for crypt counting under the microscope. Numbers of crypts required for further culture were centrifuged at $80 \times \mathrm{g}$ $\left(3 \mathrm{~min}, 4^{\circ} \mathrm{C}\right.$ ) and the pellet was resuspended in Matrigel (BD Biosciences) or in Basement Membrane Extract (BME) (Type II, R\&D). A total of 200-300 crypts were seeded per well in $8 \mu \mathrm{l}$ Matrigel or BME into a 96-well flat-bottom transparent cell culture plate (Sarstedt). Seeded crypts were incubated for 20 min at $37^{\circ} \mathrm{C}$ to let Matrigel and BME solidify. Then, $80 \mu \mathrm{l}$ of complete crypt culture medium per well was added dropwise (Advanced DMEM/F12, 0.1\% BSA, 2 mM L-glutamine, 10 mM HEPES, $100 \mathrm{U} /$ $\mathrm{ml}$ penicillin, $100 \mu \mathrm{g} / \mathrm{ml}$ streptomycin, $1 \mathrm{mM}$ $\mathrm{N}$-acetyl cysteine (Sigma), $1 \times$ B27 supplement, $1 \times \mathrm{N} 2$ supplement (Gibco), $50 \mathrm{ng} / \mathrm{ml} \mathrm{mEGF}$, and $100 \mathrm{ng} / \mathrm{ml}$ mNoggin (Peprotech). hR-spondin-1 was added as conditioned medium of hR-spondin-1-transfected HEK 293T cells to a final volume of $25 \%$ (v/v) crypt culture medium. Organoids were cultured at $37^{\circ} \mathrm{C}$ in a $5 \% \mathrm{CO}_{2}$ atmosphere for 3 days before cell death induction.

\section{Generation of tumoroids}

Organoids from tumors (tumoroids) were generated as described previously with slight modifications [26]. Briefly, the small intestine of $\mathrm{APC}^{\mathrm{Min} /+}$ mice was opened longitudinally. Tumors were isolated from intestinal tissue with scissors and forceps and cut into small pieces. Subsequently, tumor fragments were washed three times with ice cold $\mathrm{Ca}^{2+}$ - and $\mathrm{Mg}^{2+}$-free PBS, and incubated in digestion buffer (DMEM, 2.5\% FBS, $100 \mathrm{U} / \mathrm{ml}$ penicillin, $100 \mu \mathrm{g} / \mathrm{ml}$ streptomycin, $200 \mathrm{U} / \mathrm{ml}$ Collagenase IV, $125 \mu \mathrm{g} /$ $\mathrm{ml}$ Dispase II) for $1 \mathrm{~h}$ at $37^{\circ} \mathrm{C}, 5 \% \mathrm{CO}_{2}$. Tissue suspensions were shaken every $15 \mathrm{~min}$. After $1 \mathrm{~h}$ tumor fragments were allowed to settle for $1 \mathrm{~min}$. Subsequently, the supernatant was harvested and centrifuged at $200 \times \mathrm{g}$ for $3 \mathrm{~min}$ at room temperature. The pellet was resuspended in $5 \mathrm{ml}$ PBS and filtered through a $70 \mu \mathrm{m}$ and a $40 \mu \mathrm{m}$ cell strainer. After centrifugation $(200 \times \mathrm{g}$ for $3 \mathrm{~min}$ ) cells were resuspended in $500 \mu \mathrm{lPBS}$ and counted. Cell numbers were adjusted to $1.5 \times 10^{4}$ cells/50 $\mu \mathrm{l}$ Matrigel/BME. Complete growth medium with only $50 \mathrm{ng} /$ $\mathrm{ml}$ mEGF was added. The culture medium was changed every 4 days. Tumoroids were split according to their density, but in general every week. Thus, medium was removed and tumoroids were incubated in cold PBS for $1 \mathrm{~h}$ on ice. Subsequently, Matrigel/BME was dissociated mechanically with a pipet tip and tumoroids were resuspended in cold PBS. Then, tumoroids were centrifuged at $200 \times \mathrm{g}$ for 3 mins and the pellet was resuspended in TrypLE Express (Thermo Fisher) for $15 \mathrm{~min}$ at RT. Tumoroid fragments were then centrifuged at $350 \times \mathrm{g}$ for $3 \mathrm{~min}$ and split in a 1:4 ratio for further culture.
Culture of human intestinal organoids Human intestinal organoids were generated and cultured as described previously [27]. Frozen organoids were thawed and cultured in a mixture of $50 \%$ basal medium containing 500 ng/ml hR-spondin-1, 50 ng/ml mEGF, $100 \mathrm{ng} / \mathrm{ml}$ mNoggin, $10 \mathrm{nM}$ [Leu15]-Gastrin I, 10 mM Nicotinamide, 500 nM A83-01 (TGF $\beta$ inhibitor), $10 \mu \mathrm{M}$ SB202190 (p38/MAPK inhibitor), $10 \mu \mathrm{M}$ Y-27632 (ROCK inhibitor) and $50 \%$ Wnt3A-conditioned medium. Growth medium was replenished every second to third day, and organoids were passaged weekly.

Staining of organoids with PI \& Hoechst Intestinal organoids in Matrigel/BME were stained with $\mathrm{PI}$ and Hoechst at a final concentration of $10 \mu \mathrm{g} / \mathrm{ml}$ each. Staining solution (dyes in PBS) was directly added to culturing medium after treatment. Organoids were stained for $30 \mathrm{~min}$ at $37^{\circ} \mathrm{C}, 5 \% \mathrm{CO}_{2}$ for subsequent analysis on the plate reader or by fluorescence microscopy. Then, staining medium was removed and replenished with fresh phenol-red free medium before analysis.

\section{Fluorometric quantification of specific cell death in intestinal organoids}

Cell death was induced in organoids and cell lines as indicated. Before measurement, staining medium was replaced with fresh phenol-red free medium. Stained organoids still embedded in hydrogel (Matrigel/BME) were measured in a plate reader (Tecan M200 Pro). Measurements were taken from the top. First, the gain was set to the wells for the highest expected cell death (PI) and the lowest expected cell death (Hoechst). Then, Z-position was determined automatically from the corresponding wells and was checked for values between $1.5 \times 10^{6}$ and $1.6 \times 10^{6} \mu \mathrm{m}$. Subsequently, fluorescence was measured with 25 flashes, with an integration time of $20 \mu \mathrm{s}$. Lag and settle time were set to $0 \mathrm{~s}$. For each well, $4 \times 4$ measurements were taken with a border of $1 \mathrm{~mm}$ added around the measurement points. Excitation and emission wavelengths for PI were $535 \mathrm{~m}$ and $617 \mathrm{~nm}$, respectively, and for Hoechst 361 and $486 \mathrm{~nm}$, respectively. During the measurement, all wells were first measured for PI fluorescence and after a 30-s delay for Hoechst fluorescence. 
Calculation of $\mathrm{PI} / \mathrm{Hoechst}$ ratio \& treatment-specific organoid cell death The PI/Hoechst ratio was calculated by dividing PI by Hoechst RFUs:

$$
\frac{P I}{H} \text { ratio }=\frac{R F U(P I)}{R F U(\text { Hoechst })}
$$

Using $\mathrm{Pl} /$ Hoechst ratio, treatment specific organoid cell death was calculated:

treatment-specific organoid cell death [\%] = $\left(\frac{x(\text { sample })}{z(S T S)}\right) \times 100-y(u t)$

Each sample was divided by the mean of all staurosporine (STS)-treated organoids and resulting values multiplied by 100 . Then, mean of all untreated (ut) organoids was subtracted to set ut organoids to 0 .

\section{Determination of organoid viability \& specific organoid death using MTT reduction}

Organoid viability was assessed by 3-(4,5-dimethylthiazol-2-yl)-2,5-diphenyltetrazolium bromide (MTT) reduction as described in [24]. Briefly, after cell death induction MTT solution was added to the organoid culture to a final concentration of $500 \mu \mathrm{g} / \mathrm{ml}$ and incubated for $1 \mathrm{~h}$ at $37^{\circ} \mathrm{C}, 5 \%$ $\mathrm{CO}_{2}$. Then, medium was discarded and $20 \mu \mathrm{l}$ of $2 \%$ SDS solution in $\mathrm{H}_{2} \mathrm{O}$ was added to solubilize the hydrogel (Matrigel/BME) for $1 \mathrm{~h}$ at $37^{\circ} \mathrm{C}$. Subsequently, $80 \mu \mathrm{l}$ of DMSO was added and incubated for $1 \mathrm{~h}$ at $37^{\circ} \mathrm{C}$ to solubilize the reduced MTT. The optical density was then measured at $562 \mathrm{~nm}$ in a plate reader (Tecan M200 Pro).

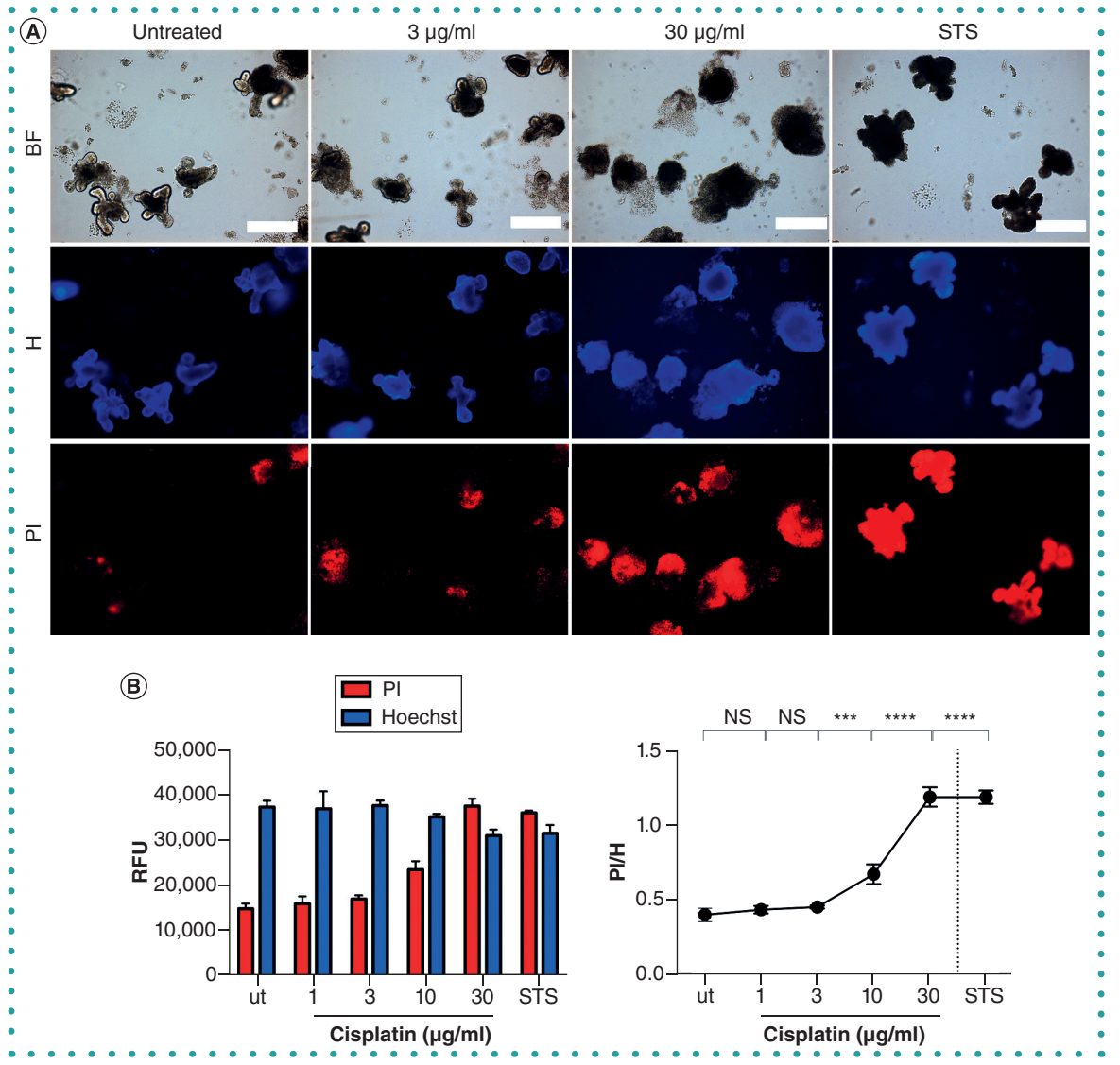

Figure 1. Quantification of $\mathrm{PI}$ and Hoechst in 3D organoids. Organoids were treated with indicated concentrations of cisplatin overnight. Then, organoids were stained with PI and Hoechst, and fluorescence was detected. (A) Representative BF and fluorescence microscopic images of untreated, $3 \mu \mathrm{g} / \mathrm{ml}$ cisplatin, $30 \mu \mathrm{g} / \mathrm{ml}$ cisplatin, and STS $(5 \mu \mathrm{M})$ treated organoids (scale bar $=200 \mu \mathrm{m}$ ). (B) Left side: RFUs of PI and Hoechst over all concentrations. Right side: ratio of PI/H over the whole dose response. Mean \pm standard deviation; $\mathrm{n}=3$ with technical triplicates. $\star * \star p \leq 0.001 ; * \star * \star p<<0.0001$.

BF: Brightfield; H: Hoechst; NS: Nonsignificant; PI: Propidium iodide; RFU: Relative fluorescence unit; STS: Staurosporine.

\section{Quantification of intracellular ATP}

Intracellular ATP was quantified with the CellTiter-GLo ${ }^{\circledR}$ 3D cell viability assay according to manufacturer's protocol
(Promega). Briefly, after treatment medium was removed and cells were lysed in $100 \mu \mathrm{l}$ pre-warmed CellTiter-GLo 3D reagent. Then, samples were incubated for $30 \mathrm{~min}$ on an
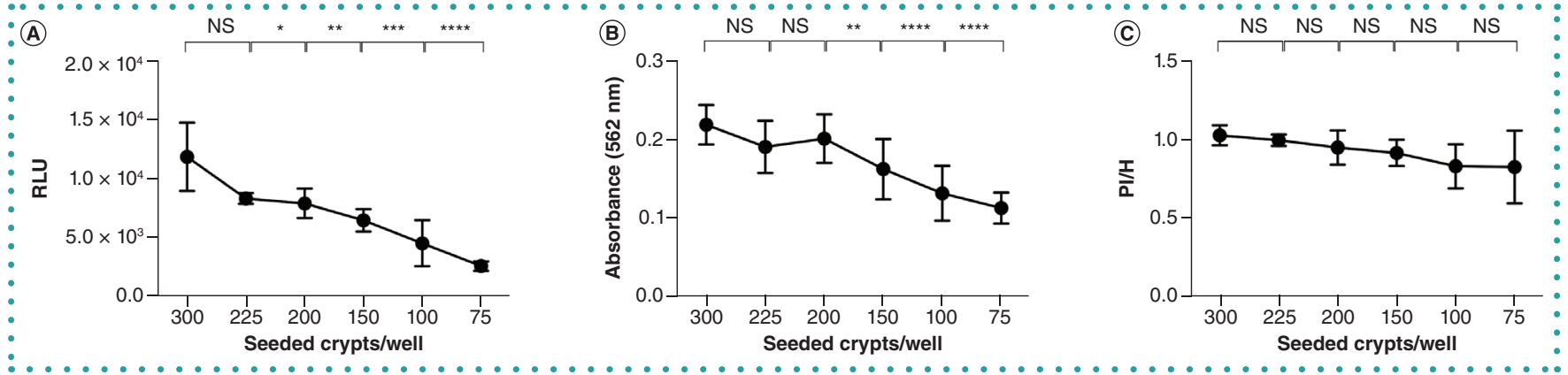

Figure 2. Assay comparison with different organoids densities. Murine intestinal crypts were seeded in indicated concentrations and grown for 3 days into organoids. Then, organoids were analyzed using CellTiterGlo (A) or MTT reduction assay (B). For PI/H fluorescence quantification (C), organoids were treated with $10 \mu \mathrm{g} / \mathrm{ml}$ cisplatin overnight before measurement.

${ }^{*} \mathrm{p} \leq 0.05$; $* * \mathrm{p} \leq 0.01 ; * * * \mathrm{p} \leq 0.001$; ****p $<0.0001$.

NS: Nonsignificant; PI/H: Propidium iodide and Hoechst; RLU: Relative luminescence unit. 

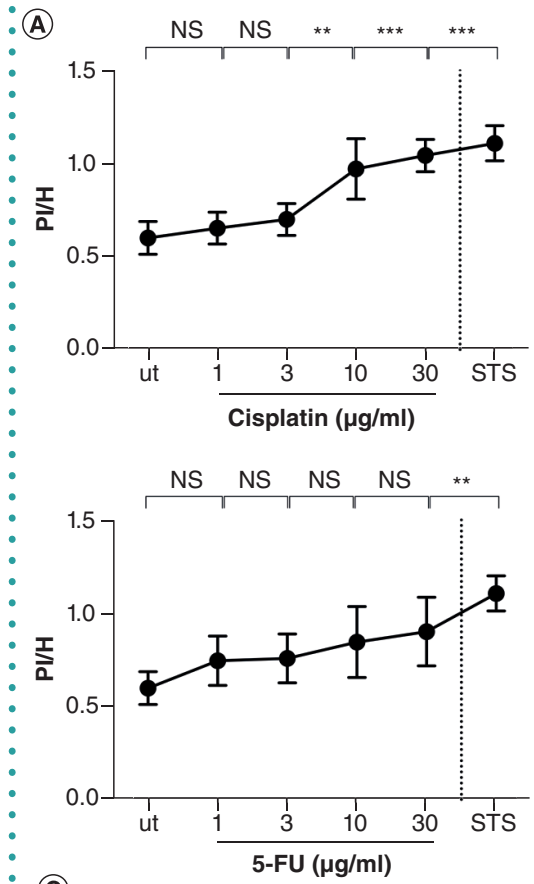

(C)

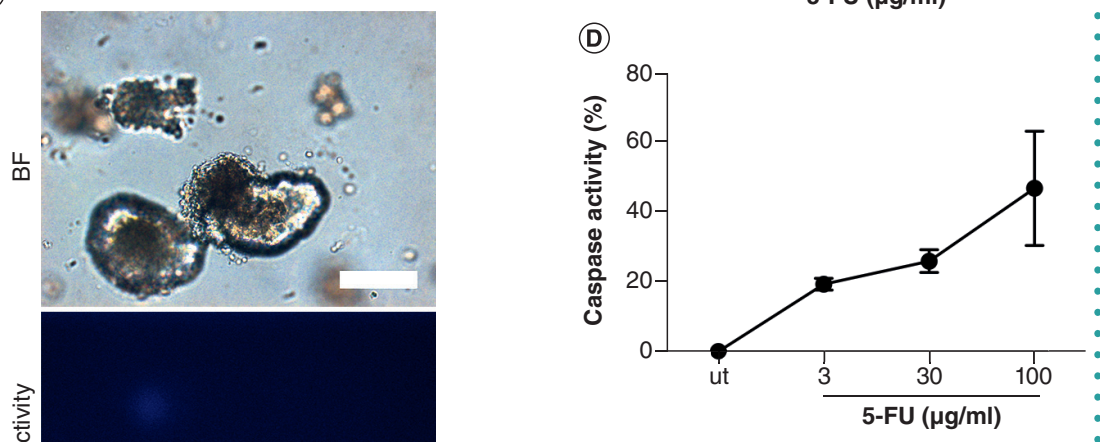

(E)

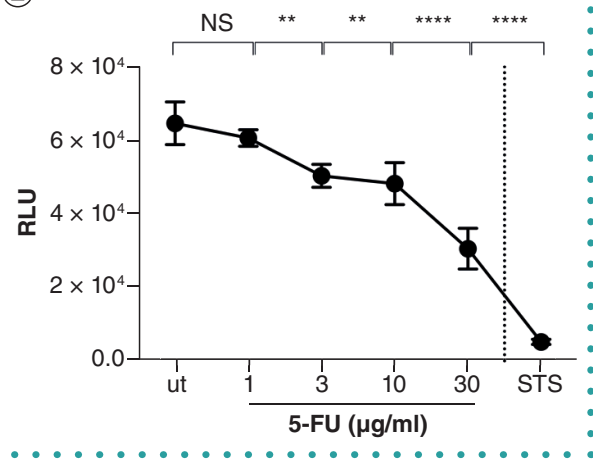

Figure 3. Propidium iodide and Hoechst quantification to calculate specific organoid cell death. Organoids were treated with indicated concentrations of chemotherapeutics and STS $(5 \mu \mathrm{M})$ overnight. Then, $\mathrm{PI} / \mathrm{H}$ ratio and treatment-specific organoid cell death were calculated (A \& $\mathrm{B})$. (C) Fluorescence image of organoid treated with $10 \mu \mathrm{g} / \mathrm{ml}$ cisplatin overnight and then stained with $\mathrm{PI}$ and Caspase $3 / 7$ substrate. (D) Caspase activity was calculated with signal of Caspase $3 / 7$ substrate. (E) RLUs, illustrating intracellular ATP-content, assessed with CellTiterGlo (scale bar $=85 \mu \mathrm{m}$ ). Mean \pm standard deviation; $\mathrm{n}=3$ with technical triplicates.

$* \mathrm{p} \leq 0.05 ; * * \mathrm{p} \leq 0.01 ; * * * \mathrm{p} \leq 0.001 ; * * * * \mathrm{p}<0.0001$.

5-FU: 5-fluorouracil; BF: Brightfield; H: Hoechst; PI: Propidium iodide; RLU: Relative luminescence unit; STS: Staurosporine. orbital shaker and luminescence was recorded afterwards using a plate reader (Tecan M200 Pro).

\section{Analysis of activated effector caspases} Activation of effector caspases 3 and 7 was performed with CellMeter"' Live Cell Caspase 3/7 Assay Kit (blue fluorescence) according to manufacturer's protocol (AAT Bioquest). In brief, organoids were stained with ApoBrite $\mathrm{U} 470$ Caspase $3 / 7$ substrate for $2 \mathrm{~h}$ at $37^{\circ} \mathrm{C}$ before treatment. Then, cell death was induced and organoids stained additionally with PI. Subsequently, fluorescence was recorded microscopically (Zeiss Axio Observer.Z1), and quantitatively at $380 \mathrm{~nm}$ (ApoBrite) and $617 \mathrm{~nm}$ (PI) using a plate reader (Tecan M200 Pro).

\section{Fluorescence microscopy}

Intestinal organoids were stained with nuclear dyes as described above and subsequently analyzed in hydrogel (Matrigel/BME) on a Zeiss Axio Observer.Z1 microscope. Brightfield images were taken with Palm-ROBO and fluorescence pictures with AxioVision Software (Zeiss).

\section{Statistical analysis}

Statistical analysis was performed using GraphPad Prism (GraphPad Prism Software, Inc.). Unless denoted otherwise, experiments were repeated three times with technical triplicates. One-way ANOVA with Dunnett's multiple comparisons test was performed.

\section{RESULTS \& DISCUSSION}

Cell death analysis in intestinal organoids in general, and its detection by $\mathrm{PI} / \mathrm{Hoechst}$ staining in particular, requires precise experimental timing. If organoids are grown for prolonged periods of time dead cells accumulate in the lumen leading to a strong PI background. Therefore, all experiments shown were performed at day 3 after crypt isolation, whereas thawed human organoids were analyzed at day 3 after splitting. Moreover, cell death induction was performed overnight to ensure proper cell membrane disintegration and nuclear staining, independent of the mode of cell death. Initially, organoids were stained for various amounts of time (5-60 $\mathrm{min})$ to assess the optimal duration of staining with both dyes. As stainings with both dyes were 
close to completion at $30 \mathrm{~min}$, this time point was used for all subsequent experiments (Supplementary Figure 1).

Figure 1A shows exemplified pictures of murine intestinal organoids treated with the chemotherapeutic drug cisplatin or the pan-kinase inhibitor STS as a positive control. Whereas the PI signal gradually increased with increasing cisplatin concentrations, the Hoechst signal was only slightly attenuated (Figure 1A \& B). Quantification of $\mathrm{PI}$ and Hoechst fluorescence enabled ratio formation, thus normalizing dying/ dead organoids (PI-positive) to total DNA (Hoechst-positive). This ratio is significantly different between untreated organoids and increasing cisplatin concentrations, or STS (Figure 1C). The internal normalization by Hoechst staining stabilized measurements over a wide range of organoid densities (Figure 2B, right), whereas other established methods assessing cellular respiratory potential revealed a strong dependency on cell numbers (Figure 2A \& B).

Having established that the ratio of $\mathrm{Pl} /$ Hoechst signal gradually increased with increasing cell death induced by increasing cisplatin concentrations (Figure 1B), we next aimed at assessing treatmentspecific organoid cell death in response to the chemotherapeutic drugs cisplatin and 5-fluorouracil (5-FU) (Figure 3A \& B). Increasing concentrations of either chemotherapeutic drug resulted in an increased $\mathrm{Pl} /$ Hoechst ratio, and an increase in the calculated treatment-specific organoid death (Figure 3A \& B). To assess whether cell death observed was associated with apoptosis, caspase activity was analyzed in parallel with $\mathrm{PI}$ staining, demonstrating double-positive cells (Figure $3 \mathrm{C}$ ) and a dose-dependent increase in caspase activity (Figure 3D). Currently, organoid cell death is frequently assessed indirectly by a reduction in intracellular ATP levels $[28,29]$. The direct comparison revealed that PI/ Hoechst staining detects chemotherapyinduced organoid cell death as sensitively as intracellular ATP levels (Figure 3B \& E).

In order to verify that cell death analysis by $\mathrm{PI} /$ Hoechst staining is not limited to murine primary intestinal organoids, we also assessed cell death quantification with $\mathrm{Pl} / \mathrm{Hoech}$ st staining in murine tumoroids (Figure 4A-C) and human intestinal organoids (Figure 4D), confirming the

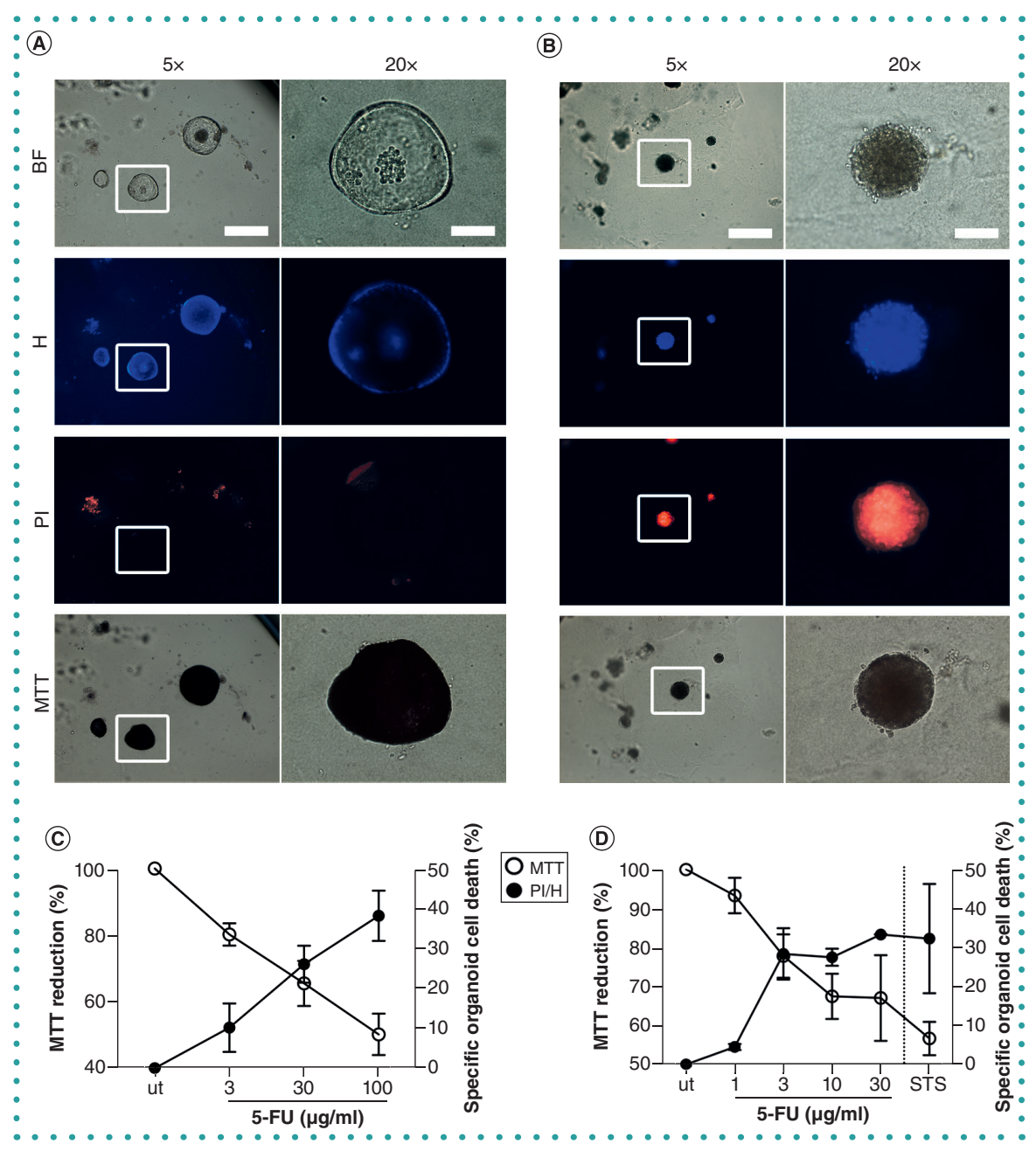

Figure 4. Multiplexing $\mathrm{PI} / \mathrm{H}$ quantification with $\mathrm{MTT}$-reduction assay. Tumoroids from $\mathrm{APC}^{+/-}$mice were treated with indicated concentrations of 5-FU overnight, stained with $\mathrm{PI} / \mathrm{H}$, and fluorescence was quantified. Subsequently, MTT solution was added and MTT reduction was measured. BF and fluorescence microscopic images of untreated tumoroid (A) and 5-FU treated tumoroid (B). (C) $\mathrm{PI} / \mathrm{H}$ fluorescence derived specific organoid cell death (right axis), MTT reduction (left axis). (D) Human organoids treated with cisplatin. $\mathrm{PI} / \mathrm{H}$ ratio and MTT reduction were measured, and specific organoid cell death as well as MTT reduction were calculated thereof. Scale bar $(5 x)=350 \mu \mathrm{m}$, $(20 x)=85 \mu \mathrm{m}$. Mean \pm standard deviation; $\mathrm{n}=3$ with technical triplicates.

5-FU: 5-fluorouracil; BF: Brightfield; PI/H: Propidium iodide and Hoechst.

suitability of this method for other types of organoids.

We next set to multiplex cell death assessment by combining $\mathrm{PI} /$ Hoechst staining with our previously established method of measuring organoid viability by MTT reduction [30]. Therefore, organoids from intestinal tumors of APC ${ }^{\text {min }}$ mice (tumoroids) were treated with indicated concentrations of 5-FU, stained with $\mathrm{PI}$ and Hoechst, and fluorescence was measured. Subsequently, PI/Hoechststained tumoroids were further incubated with MTT and reduction capacity was analyzed by absorbance of the resulting formazan at $562 \mathrm{~nm}$. Whereas viable (ut) tumoroids efficiently reduced MTT to purple formazan (Figure 4A), tumoroids treated with 5-FU showed morphological disintegration and failed to reduce MTT (Figure 4B). $A$ decrease in MTT reduction was paralleled by an increase in PI signal, but stable Hoechst staining (Figure 4A \& B). Quantification of $\mathrm{PI} / \mathrm{Hoech}$ st fluorescence and formazan absorption enabled calculation of treatment-specific organoid cell death (PI/Hoechst), which inversely correlated with decreased MTT reduction (Figure 4C). Multiplexing $\mathrm{PI} /$ Hoechst staining and MTT reduction was also confirmed by analyzing cisplatin-induced cell death in human intestinal organoids (Figure 4D). These data 
demonstrate that analysis of cell death by $\mathrm{PI} /$ Hoechst and cell survival by MTT reduction can be combined in $3 \mathrm{D}$ organoid cultures.

In conclusion, we here present a fast and simple method to quantify organoid cell death by measuring $\mathrm{PI}$ and Hoechst fluorescence in $3 \mathrm{D}$ in a plate reader. The method is cheap, reliable, and does not require commercially available kits, or complicated and time-consuming high content imaging analysis $[21,25]$. Thus, it is applicable for analysis of treatment-specific organoid death without the need to invest in expensive equipment or bioinformatics. By multiplexing this method with assays assessing respiratory changes, such as MTT reduction [30], information gain is increased and might help to distinguish between direct cell death-inducing agents and inhibitors of cellular respiration and metabolism.

\section{FUTURE PERSPECTIVE}

Organoid technology is already being used to investigate patient-specific drug response. Usage of patient-derived organoids is likely toincrease in the future. Thus, fast and robust methods are needed to quantitatively assess cell death in organoids. As our method is fast and simple it can be used frequently in the future to quickly assess death-inducing effects of drugs in PDOs.

\section{SUPPLEMENTARY DATA}

To view the supplementary data that accompany this paper please visit the journal website at: www.future-science. com/doi/suppl/10.2144/btn-2019-0023

\section{ACKNOWLEDGMENTS}

$\mathrm{APC}^{\mathrm{Min} /+}$ mice were a kind gift of Jan Paul Medema, Amsterdam, The Netherlands.

\section{AUTHOR CONTRIBUTIONS}

KJB designed the study, conducted most experiments, and wrote the manuscript, SM conducted some experiments and contributed to the manuscript, MS and MM gave technical advice, refined the manuscript and provided human jejunal cells, TB designed and supervised the study, provided funding and finalized the manuscript.

\section{AUTHOR CONTRIBUTIONS}

$\mathrm{APC}^{\mathrm{Min} /+}$ mice were a kind gift from Jan Paul Medema, Amsterdam, The Netherlands.
FINANCIAL \& COMPETING INTERESTS DISCLOSURE

KJ Bode was supported by a fellowship from the Baden-Württemberg Ministry of Science, Research and Art-funded Co-operative research training school 'Advanced in vitro test systems for the analysis of cell-chemical interactions in drug discovery and environmental safety' (InViTe). The authors have no other relevant affiliations or financial involvement with any organization or entity with a financial interest in or financial conflict with the subject matter or materials discussed in the manuscript apart from those disclosed.

No writing assistance was utilized in the production of this manuscript.

\section{ETHICAL CONDUCT OF RESEARCH}

The authors state that they have obtained appropriate institutional review board approval or have followed the principles outlined in the Declaration of Helsinki for all human or animal experimental investigations. In addition, for investigations involving human subjects, informed consent has been obtained from the participants involved.

\section{OPEN ACCESS}

This work is licensed under the AttributionNonCommercial-NoDerivatives 4.0 Unported License. To view a copy of this license, visit http://creativecommons.org/licenses/ by-nc-nd/4.0/

\section{REFERENCES}

Papers of special note have been highlighted as: • of interest

1. Sato T, Vries RG, Snippert HJ et al. Single Lgr5 stem cells build crypt-villus structures in vitro without a mesenchymal niche. Nature 459(7244), 262-265 (2009).

- Murine intestinal organoids are described for the first time.

2. Marx V. Tissue engineering: organs from the lab. Nature 522(7556), 373-377 (2015).

3. Clevers $\mathrm{H}$. Modeling development and disease with organoids. Cell 165(7), 1586-1597 (2016).

4. Meneses AMC, Schneeberger K, Kruitwagen HS et al. Intestinal organoids - current and future applications. Vet. Sci. 3(4), (2016).

5. Lancaster MA, Knoblich JA. Organogenesis in a dish: modeling development and disease using organoid technologies. Science 345(6194), 1247125 (2014).

6. Yu H, Hasan NM, In JG et al. The contributions of human mini-intestines to the study of intestinal physiology and pathophysiology. Annu. Rev. Physiol. 79, 291-312 (2017).

7. Howell KJ, Kraiczy J, Nayak KM et al. DNA methylation and transcription patterns in intestinal epithelial cells from pediatric patients with inflammatory bowel diseases differentiate disease subtypes and associate with outcome. Gastroenterology 154(3), 585-598 (2017).

8. Grabinger T, Bode KJ, Demgenski J et al. Inhibitor of apoptosis protein-1 regulates tumor necrosis factor-mediated destruction of intestinal epith
Gastroenterology 152(4), 867-879 (2017).

Murine and human intestinal organoids are used to investigate the interplay of TNF and cIAP1 in the intestine.
9. In JG, Foulke-Abel J, Estes MK, Zachos NC, Kovbasnjuk 0 , Donowitz M. Human mini-guts: new insights into intestinal physiology and host-pathogen interactions. Nat. Rev. Gastroenterol. Hepatol. 13(11), 633 (2016).

10. Yui S, Nakamura T, Sato T et al. Functional engraftment of colon epithelium expanded in vitro from a single adult Lgr5(+) stem cell. Nat. Med. 18(4), 618-623 (2012).

11. Lee SH, Hu W, Matulay JT et al. Tumor evolution and drug response in patient-derived organoid models of bladder cancer. Cell 173(2), 515-528 e517 (2018).

12. Roerink SF, Sasaki N, Lee-Six H et al. Intra-tumour diversification in colorectal cancer at the single-cell level. Nature 556(7702), 457-462 (2018).

13. Ranga A, Gjorevski N, Lutolf MP. Drug discovery through stem cell-based organoid models. Adv. Drug Deliv. Rev. 69-70, 19-28 (2014)

14. Astashkina A, Grainger DW. Critical analysis of 3-D organoid in vitro cell culture models for high-throughput drug candidate toxicity assessments. Adv. Drug Deliv. Rev. 69-70, 1-18 (2014)

15. Bulin AL, Broekgaarden $M$, Hasan T. Comprehensive high-throughput image analysis for therapeutic efficacy of architecturally complex heterotypic organoids. Sci. Rep. 7(1), 16645 (2017).

16. Kondo J, Ekawa $\mathrm{T}$, Endo $\mathrm{H}$ et al. High-throughput screening in colorectal cancer tissue-originated spheroids. Cancer Sci. 110(1), 345 (2018).

17. Gao D, Vela I, Sboner A et al. Organoid cultures derived from patients with advanced prostate cancer. Cell 159(1), 176-187 (2014).

18. Huang L, Holtzinger A, Jagan I et al. Ductal pancreatic cancer modeling and drug screening using human pluripotent stem cell- and patient-derived tumor organoids. Nat. Med. 21(11), 1364-1371 (2015).

19. Hubert CG, Rivera M, Spangler LC et al. A three-dimensional organoid culture system derived from human glioblastomas recapitulates the hypoxic gradients and cancer stem cell heterogeneity of tumors found in vivo. Cancer Res. 76(8), 2465-2477 (2016).

20. Perkhofer L, Frappart PO, Muller M, Kleger A. Importance of organoids for personalized medicine. Per. Med. 15(6), 461-465 (2018).

21. Vlachogiannis G, Hedayat $S$, Vatsiou A et al. Patient-derived organoids model treatment response of metastatic gastrointestinal cancers. Science 359(6378), 920-926 (2018).

Patient-derived organoids are used as preclinical models.

22. Wills ES, Drenth JP. Building pancreatic organoids to aid drug development. Gut. 66(3), 393-394 (2017).

23. Saeed K, Rahkama V, Eldfors $S$ et al. Comprehensive drug testing of patient-derived conditionally reprogrammed cells from castration-resistant prostate cancer. Eur. Urol. 71(3), 319-327 (2017).

24. Grabinger T, Luks L, Kostadinova $\mathrm{F}$ et al. Ex vivo culture of intestinal crypt organoids as a model system for assessing cell death induction in intestinal epithelial cells and enteropathy. Cell Death Dis. 5, e1228 (2014).

25. Jabs J, Zickgraf FM, Park J et al. Screening drug effects in patient-derived cancer cells links organoid responses to genome alterations. Mol. Syst. Biol. 13(11), 955 (2017).

- The authors use the nuclear dyes Hoechst and propidium iodide to microscopically assess organoid cell death.

26. Xue X, Shah YM. In vitro organoid culture of primary mouse colon tumors. J. Vis. Exp. 75, e50210 (2013).

27. Schweinlin M, Wilhelm S, Schwedhelm I et al. Development of an advanced primary human in vitro model of the small intestine. Tissue Engineering Part C: Methods. 22(9), 873-883 (2016)

28. Boehnke K, Iversen PW, Schumacher D et al. Assay establishment and validation of a high-throughput screening platform for three-dimensional patient-derived colon cancer organoid cultures. J. Biomol. Screen. 21(9), 931-941 (2016).

29. Francies HE, Barthorpe A, McLaren-Douglas A, Barendt WJ, Garnett MJ. Drug sensitivity assays of human cancer organoid cultures. Methods Mol. Biol. 1-12 (2016).

- Organoids are employed for a drug sensitivity assay using a commercial kit detecting changes in intracellular ATP.

30. Grabinger T, Delgado E, Brunner T. Analysis of cell death induction in intestinal organoids in vitro. Methods Mol. Biol. 1419, 83-93 (2016).

The authors describe how MTT reduction in intestinal organoids can be used to assess cell death. 